\title{
Direct generation of local orbitals for multireference treatment and subsequent uses for the calculation of the correlation energy
}

\author{
Daniel Maynau ${ }^{\mathrm{a})}$ \\ Laboratoire de Physique Quantique UMR 5626 du CNRS, IRSAMC, Université Paul Sabatier, 118, \\ route de Narbonne, 31062 Toulouse, Cedex, France \\ Stefano Evangelisti \\ Laboratoire de Physique Quantique UMR 5626 du CNRS, IRSAMC, Université Paul Sabatier, 118, \\ route de Narbonne, 31062 Toulouse Cedex, France and Dipartimento di Chimica Fisica e Inorganica, \\ Università di Bologna-Viale Risorgimento 4, I-40136 Bologna, Italy \\ Nathalie Guihéry \\ Laboratoire de Physique Quantique UMR 5626 du CNRS, IRSAMC, Université Paul Sabatier, 118, \\ route de Narbonne, 31062 Toulouse, Cedex, France
}

Carmen J. Calzado

Laboratoire de Physique Quantique UMR 5626 du CNRS, IRSAMC, Université Paul Sabatier, 118, route de Narbonne, 31062 Toulouse, Cedex, France and Departamento de Quimica Fisica, Universidad de Sevilla-c/bi Professor Garcia Gonzalez, s/n. E-41012, Sevilla, Spain

Jean-Paul Malrieu

Laboratoire de Physique Quantique UMR 5626 du CNRS, IRSAMC, Université Paul Sabatier, 118, route de Narbonne, 31062 Toulouse, Cedex, France

(Received 7 November 2001; accepted 15 March 2002)

\begin{abstract}
We present a method that uses the one-particle density matrix to generate directly localized orbitals dedicated to multireference wave functions. On one hand, it is shown that the definition of local orbitals making possible physically justified truncations of the CAS (complete active space) is particularly adequate for the treatment of multireference problems. On the other hand, as it will be shown in the case of bond breaking, the control of the spatial location of the active orbitals may permit description of the desired physics with a smaller number of active orbitals than when starting from canonical molecular orbitals. The subsequent calculation of the dynamical correlation energy can be achieved with a lower computational effort either due to this reduction of the active space, or by truncation of the CAS to a shorter set of references. The ground- and excited-state energies are very close to the current complete active space self-consistent field ones and several examples of multireference singles and doubles calculations illustrate the interest of the procedure. (C) 2002 American Institute of Physics. [DOI: 10.1063/1.1476312]
\end{abstract}

\section{INTRODUCTION}

The Lewis representation of molecules in terms of bonds and lone pairs of electrons commonly used in chemistry is probably the first example of a relevant local description. Before the success of Koopmans' theorem ${ }^{1}$ induced a prevalence of the delocalized treatments, several localized methods providing interesting physical analysis were proposed. The valence bond method, ${ }^{2}$ which gives a physically motivated hierarchy of the determinants in terms of neutral, singly ionic, doubly ionic, etc., is one of the best examples of such treatments. Later on, it was shown ${ }^{3}$ for single reference (single determinantal) problems that both local and nonlocal descriptions are equivalent, since one may always define a unitary transformation to go from the delocalized occupied orbitals to the localized occupied ones so that a single reference is preserved. The Fock operator, which is diagonal in the canonical set, becomes block diagonal, i.e., the elements between occupied and virtual molecular orbitals (MO) re-

${ }^{a)}$ Electronic-mail: daniel.maynau@irsamc.ups-tlse.fr main null, according to Brillouin's theorem. ${ }^{4}$ The equivalence of these descriptions may be extended to multireference (CAS) problems, provided that the transformations are only made inside the different blocks of inactive, active, and virtual orbitals.

The more recent growth of interest for localized descriptions was initially due to the possible neglect of small enough bielectronic integrals when localized orbitals are used, and then widely motivated by the calculation of the correlation energy for a lower computational effort. Indeed, since the number of virtual orbitals needed for the correlation of each electron pair may be dramatically reduced in a local description, significant savings of CPU time could also be obtained for the correlated methods. In that philosophy, several local correlated treatments have recently been proposed, the computational cost of which scales linearly with the molecular size. Among the most important ones, one may cite the Møller-Plesset perturbation theory (MP2, MP3, MP4), ${ }^{5-11}$ the singles and doubles configuration interaction (CISD) ${ }^{12}$ and singles and doubles coupled cluster 
$(\mathrm{CCSD})^{12,13}$ and $\operatorname{CCSD}(\mathrm{T}) \cdot{ }^{14}$ All these proposals are single reference methods. However, the benefit of a local description for the calculation of the correlation energy is not an attribute singular to the single reference description. One may in fact reduce the computational effort for multireference descriptions too, when using a set of localized orbitals.

In several problems requiring multireference descriptions, the active orbitals are often concentrated in a local region. This is the case, for instance, when we consider bond breaking, magnetic systems with singly occupied orbitals localized on metal atoms, as well as spectroscopic problems that imply local excitations. An accurate description of such systems generally requires highly correlated treatments that have been, up to now, only accessible for the smaller ones. The interest of a local picture is twofold.

The first advantage is qualitative, and concerns the nature and the size of the active space. Let us consider, for instance, the case of a bond breaking. In a delocalized description, the active orbitals are the ones that bring the largest correlation energy; therefore, there is no control over their spatial location. Using local active orbitals, on the contrary, it becomes possible to restrict them to those that are actually broken during the chemical reaction.

(ii) The second advantage concerns the possible reduction of the size of the reference space. The use of complete active space (CAS) of canonical MOs is responsible for a very steep increase of the number of references included in the calculation. This may be easily understood when considering localized active orbitals obtained from the delocalized ones by unitary transformations, for instance. The isodimensional reference space generated by the CAS (now in localized orbitals) contains determinants in which all the electrons are concentrated in a certain region of space (highly ionic in the VB sense), and therefore whose weight in the wave function is close to zero. As shown in Ref. 15 , a localized multireference description instead of the CAS would be both physically justified and significantly less expensive. It is then intuitively clear that the definition of localized active orbitals combined with an appropriate multireference space would be more effective for the treatment of these problems. Although this work will not address the question of $\mathrm{N}$-scaling behavior of the calculations, let us notice that the use of a complete set of local orbitals allows the possible neglect of small enough bielectronic integrals as well as the local treatment of electron correlation.

The method uses the one-particle density matrix calculated from the $\mathrm{CI}$ of the single excitations acting on the multireference space. It will be presented in the second section. In the third section, different applications illustrate the efficiency of the proposed density matrix based multiconfiguration method in localized orbitals. The energies obtained from CASCI (configuration interaction inside the CAS) calculations carried out using the obtained set of localized orbitals are very close to the current complete active space self- consistent field (CASSCF) ones, for the ground state as well as for the excited ones. Calculations at the MR $+\mathrm{SD}$ (multireference singles and doubles) level have been performed for several chemical systems, and a systematic comparison between the results obtained for truncated and nontruncated CI calculations will be presented. Different outlooks for taking advantage of the localized description in the scaling of the correlation energy calculations are discussed in the Conclusion.

\section{PRESENTATION OF THE METHOD}

We propose an a priori method. It may be decomposed in two steps. The first one provides an initial guess of localized orbitals that will then be optimized in a second step. They may be obtained through different procedures provided that the orbitals are strongly localized and define an orthogonal set.

\section{A. What kind of local orbitals?}

The molecular orbitals commonly used in standard chemistry calculations are obtained through self-consistent field (SCF) or CASSCF procedures.

It is possible to replace the occupied (respectively, unoccupied) orbitals by a linear combination of themselves without changing the SCF energy. As well as in the canonical SCF orbitals, the choice of orbitals localized on bonds enables one to build a single determinant wave function. Notice that not only covalent bonds are concerned; it is also possible to build core or lone pair orbitals in the same way. In that case, the orbitals are atom centered and doubly occupied.

It is also possible to use singly occupied atom-centered orbitals, like the two 1s orbitals of the $\mathrm{H}_{2}$ molecule, for example. The VB tradition uses such orbitals. The generalized valence bond $(\mathrm{GVB})^{16}$ method variationally optimizes valence orbitals centered on a given atom with appropriate tail. Using singly occupied atom-centered orbitals, it is not possible to build a single determinant which gives the SCF energy. However, as will be shown later on, this can be an interesting solution. In the case of quasidegenerate systems, for instance, a correct description can be obtained only through a CI calculation involving several determinants. If the CI space is very large, it may be necessary to truncate it and perform a selected CI. In this case, the size of the selected space required to have a correct result will not be the same depending on the considered orbitals, as will be shown in the examples of Sec. III.

In this work, we develop a formalism that permits all the above possibilities. The orbitals can be localized on a single atom (for example, a core orbital or a lone pair), or a group of atoms (a bond or a fragment such as a complete aromatic ring, for instance).

\section{B. A priori methods: The generation of localized SCF orbitals}

When looking for a localization method, the determination of localized Hartree-Fock (HF) molecular orbitals (MOs) seems to be a rational choice, as far as single reference problems are concerned. Several methods are using $a$ posteriori relocalizing unitary transformations, for instance 
TABLE I. Butadiene molecule. Occupation of the local bond orbitals of the single $\mathrm{C}_{1} \mathrm{C}_{2}$ and the double $\mathrm{C}_{1} \mathrm{C}_{3}$ bonds. The $\sigma$ bonding and $\sigma^{*}$ antibonding orbitals clearly appear in both bonds with occupations close to zero. The same applies for the $\pi$ and $\pi^{*}$ orbitals in the double bond. The delocalization of these $\pi$ orbitals on the single bonds is illustrated by the $\pi$ occupations of 1.28 and 0.66 on the single bond. The other orbitals (h) are hybrid orbitals (see the text).

\begin{tabular}{lcccccccc}
\hline \hline \multicolumn{8}{c}{$\mathrm{C}_{1} \mathrm{C}_{2}$ (single bond) } \\
\hline Occupation & 1.95 & 1.28 & $\begin{array}{c}1.22 \\
\mathrm{~h}\end{array}$ & $\begin{array}{c}1.04 \\
\mathrm{~h}\end{array}$ & $\begin{array}{c}1.01 \\
\mathrm{~h}\end{array}$ & $\begin{array}{c}0.92 \\
\mathrm{~h}\end{array}$ & $\begin{array}{c}0.66 \\
\pi^{*}\end{array}$ & $\begin{array}{c}0.02 \\
\sigma^{*}\end{array}$ \\
Orbitals & $\sigma$ & $\pi$ & \multicolumn{7}{c}{$\begin{array}{c}\mathrm{C}_{1} \mathrm{C}_{3} \text { (double bond) } \\
\text { Occupation }\end{array}$} & 1.96 & 1.93 & $\begin{array}{c}1.25 \\
\mathrm{~h}\end{array}$ & $\begin{array}{c}1.15 \\
\mathrm{~h}\end{array}$ & $\begin{array}{c}0.99 \\
\mathrm{~h}\end{array}$ & $\begin{array}{c}0.92 \\
\mathrm{~h}\end{array}$ & $\begin{array}{c}0.05 \\
\pi^{*}\end{array}$ & $\begin{array}{c}0.03 \\
\sigma^{*}\end{array}$ \\
\hline \hline
\end{tabular}

the methods of Boys, ${ }^{17}$ Edminston and Ruedenberg, ${ }^{18}$ Pipek, ${ }^{19}$ and Angeli et al.,${ }^{20}$ which define an intrinsic criterion of localization (i.e., they maximize a localization function). Relocalizations that use an extrinsic criterion ${ }^{3}$ of localization (such as projecting localized MOs on the canonical ones) work equally well.

A direct way to obtain localized orbitals, i.e., one that avoids the passage through the determination of canonical MOs, was opened by the a priori methods. Some of them use a localization potential in the SCF equations. ${ }^{21-23}$ Other methods start from guess-localized orbitals and use a procedure of orbital optimization that keeps their local nature. As well as in delocalized descriptions, the optimization process can be based on an energy minimization or use the oneparticle density matrix. While in the delocalized descriptions the density matrix is directly diagonalized, giving the delocalized natural orbitals, keeping the local character of the orbitals requires avoidance of such diagonalization and control of the rotations between the orbitals.

\section{Determination of guess of local orbitals}

The set of chosen atomic orbitals $\left\{\chi_{l}\right\}$ should preferably be of ANO (atomic natural orbital) type. ${ }^{24}$ In this way, the occupied and antibonding molecular orbitals have large coefficients on the minimal basis set only. It is constituted of core orbitals, valence orbitals, and some additional nonvalence orbitals for extended basis calculations. Through the following procedure, we will build orthogonal atomic orbitals (OAO) in a first step, and localized MOs (LMOs) which are linear combinations of the OAOs. In the present approach, a great flexibility is permitted to the localized orbitals. An LMO can be atom centered for lone pairs or core (in that case, it is equal to an OAO), or bond centered, or even delocalized on a molecular fragment. These orbitals are distributed in the two classes (occupied and virtual).

The procedure starts by the generation of OAO through a hierarchical orthogonalization of the atomic orbitals that does not mix the different types (core, valence, and additional nonvalence) of orbitals. One uses a hierarchical blockSchmidt orthogonalization scheme, where the usual normalization step is replaced by an $S^{-1 / 2}$ orthogonalization inside each block corresponding to a different type of orbital. $S$ being the overlap matrix, the core orbitals that must be kept pure for an accurate guess have the highest priority, so they will just be orthogonalized among themselves using an $S^{-1 / 2}$ method.

The valence orbitals are orthogonalized to the core ones by projecting out the core components and, then, among themselves by an $S^{-1 / 2}$ orthogonalization. Finally, the additional nonvalence orbitals receive the same treatment as the valence ones (they are orthogonalized to the two previous sets) and the complete set of OAO $\left\{\tilde{\chi}_{l}\right\}$ is obtained.

We want now to obtain LMOs that correctly describe bonds or molecular fragments. The method proceeds through the calculation of a mono-electronic (SCF, Huckel...) density matrix, which was already used in the past to determine atom-centered orbitals appropriate for nondynamical correlation calculations. ${ }^{25,26}$ The density matrix is then expressed in the OAOs basis $\left\{\tilde{\chi}_{l}\right\}$

$$
R_{i j}=\left\langle\Phi_{0}\left|a_{i}^{+} a_{j}\right| \Phi_{0}\right\rangle \text {. }
$$

Let us introduce an orthogonal projector on an atom $K$ of the molecule as being the sum of the projectors on its OAOs (this definition is commonly used ${ }^{19}$ )

$$
P_{K}=\sum_{l \in K}\left|\tilde{\chi}_{l}\right\rangle\left\langle\tilde{\chi}_{l}\right|
$$

A projector on a fragment $F$ (for instance, two bonded atoms if one looks for a bond LMO) may be defined as the sum of the projectors on the atoms belonging to that fragment

$$
P_{F}=\sum_{K \in F} P_{K}
$$

The density matrices calculated on the projected functions $P_{F} \Phi_{0}$ are then diagonalized to get a new set of LMOs. The left orbitals are distributed in the classes of occupied and virtual LMOs according to their occupation numbers (typically close to 2 and 0 , respectively). The orbitals exhibiting other occupation numbers are simply rejected.

As an example, let us follow the construction of guess orbitals for the butadiene molecule. In this simple case, the choice is quite straightforward. In addition to the core orbitals of the carbons, the bonds to be constructed correspond to the Lewis graph of the $\mathrm{C}_{4} \mathrm{H}_{6}$ molecule. From the density matrix expressed in the basis of the OAOs, partial density matrices are extracted for each bond.

Table I presents an example of generation of guess orbitals for both single and double $\mathrm{C}-\mathrm{C}$ bonds. The dimension 
of the partial density matrix is $8 \times 8$, which corresponds to the four $2 s, 2 p$ orbitals on each atom. Once diagonalized, this partial density matrix automatically gives one or two (for the single or double bond) occupied orbitals and their corresponding antibonding ones. The other eigenvalues correspond to occupations of the remaining bonds of the carbon atoms. They are hybrid orbitals which belong to other bonds, and they are discarded. On may notice that the $\pi$ bonding orbital occupation on the single bond is quite large, even if it is clearly unoccupied. This phenomenon corresponds to the delocalization of the $\pi$ cloud between the double bonds through the central one. The corresponding $\pi^{*}$ antibonding orbital has also an occupation rather far from zero.

There are six $4 \times 4$ density submatrices for the $\mathrm{C}-\mathrm{H}$ bonds. At the end, one has three $\sigma \mathrm{C}-\mathrm{C}$, six $\sigma \mathrm{C}-\mathrm{H}$, and two $\pi \mathrm{C}-\mathrm{C}$ bonds. In the $\mathrm{OAO}$ basis, the valence $s$ and $p$ orbitals of $\mathrm{C}$ and $\mathrm{H}$ are replaced by the bond $\mathrm{C}-\mathrm{C}$ and $\mathrm{C}-\mathrm{H}$ orbitals. The core and virtual OAO remain unchanged. If one wants to use atomic $p$ orbitals instead of $\pi$ bonds, it is possible to keep the $p$ OAOs and to ignore the $\pi$ and $\pi^{*}$ local orbitals. In this case, a single reference calculation is impossible.

Of course, the new basis is nonorthogonal. A new hierarchical orthogonalization is applied on these orbitals, as it was done to build the OAOs.

This procedure, after being repeated on all bonds, provides a nonorthogonal set of localized orbitals. The generation of an orthogonal space is achieved using a hierarchical orthonormalization similar to the one described for the first set of atomic orbitals. The highest priority is given to the inactive LMOs, then to the active ones, and finally to the virtual ones, each class of orbitals being orthogonalized by an $S^{-1 / 2}$ procedure.

Let us recall some advantages of the method:

Due to the use of a hierarchical orthogonalization scheme, the procedure provides an orthogonal set of strongly localized orbitals.

The method avoids the problem of relocalizing the virtual nonvalence orbitals by considering the orthogonal atomic ones.

(iii) Finally, for local symmetry reasons, a proper hybridization of the orbitals is obtained.

\section{Optimization of the localized orbitals: General approach}

The molecular orbitals $\phi_{i}$ obtained from an SCF procedure fulfill two properties.

(1) The energy $\left\langle\Phi_{0}|H| \Phi_{0}\right\rangle$ is minimal, where $\Phi_{0}$ $=\left|\phi_{1} \overline{\phi_{1}} \cdots \phi_{n} \overline{\phi_{n}}\right|, n$ being the number of occupied orbitals.

(2) The single configuration interaction (CIS) density matrix is diagonal, with eigenvalues 2 for the occupied, and 0 for virtual orbitals.

These properties are not independent. In an SCF procedure, the energy is minimized and, as a consequence, the second property is verified. One could choose to fulfill property (2) first and to obtain the SCF energy in this way. The method presented here follows this scheme and provides, among all the possible orbitals giving the SCF energy, a set of localized ones.

The procedure starts from the definition of a single reference $\Phi_{0}$ built on a set of occupied strongly localized guess orbitals that is then separated in two classes, the inactive I and the virtual $\mathrm{V}$ orbitals. Then, from the singly excited determinants $a_{r}^{+} a_{i} \Phi_{0}$, where $a_{r}^{+}$(respectively, $a_{i}$ ) is the creation (respectively, annihilation) operator of an electron in the virtual orbital $r$ (respectively, occupied $i$ ), the CIS matrix is calculated. The diagonalization of this matrix provides the coefficients of the singly excited determinants $C_{i r}$ in the $\Psi_{\text {CIS }}$ wave function, which can be written in intermediate normalization (the coefficient of the reference $\Phi_{0}$ is $C_{0}=1$ )

$$
\Psi_{\mathrm{CIS}}=\Phi_{0}+\sum_{i, r} C_{i r} a_{r}^{+} a_{i} \Phi_{0}
$$

These coefficients are then used to compute the density matrix $R$ corresponding to $\Psi_{\text {CIS }}$. The off-diagonal elements of $R$ are given by

$$
R_{i r}=\left\langle\Psi_{\mathrm{CIS}}\left|a_{r}^{+} a_{i}\right| \Psi_{\mathrm{CIS}}\right\rangle=C_{i r} .
$$

The diagonalization of $R$ gives the quasinatural orbitals (NOs) corresponding to $\Psi_{\text {CIS }}$. A new CIS performed using these NOs will give a new wave function $\Psi_{\text {CIS }}^{\prime}$, which appears to be of lower energy in all practical cases

$$
\left\langle\Psi_{\text {CIS }}|H| \Psi_{\text {CIS }}\right\rangle<\left\langle\Psi_{\text {CIS }}^{\prime}|H| \Psi_{\text {CIS }}^{\prime}\right\rangle .
$$

The new density matrix $R^{\prime}$ will have smaller offdiagonal matrix elements and the diagonal elements will be closer to 2 or 0 . At convergence, the process gives a diagonal density matrix $\left(R_{i r}=C_{i r}=0\right)$. The Brillouin theorem is verified, and the SCF energy is reached.

The diagonalization of the density matrix would delocalize the orbitals, and the original locality of the guess orbitals would be lost. One must notice, however, that if we consider a matrix diagonalization as rotation of orbitals, only the rotations between occupied and virtual are useful to converge toward a solution with HF energy. At each iteration, rotations between occupied and virtual orbitals may be frozen. This technique of partial diagonalization gives a set of localized orbitals and the energy of $\Phi_{0}$ is the SCF energy. Notice that the converged density matrix is diagonal, even if we proceed through partial diagonalizations.

The way to keep the local character of the orbitals along the iterations is similar to the one proposed in Ref. 27. Let us briefly recall the general features of this previous method. The coefficients of $\Psi_{\text {CIS }}$ are used as first-order corrections to the one electron orbitals according to the equations

$$
\begin{aligned}
& i^{\prime}=i+\sum_{\tau} C_{i r} r, \\
& r^{\prime}=r-\sum_{r} C_{i r} i,
\end{aligned}
$$

where $i$ and $r\left(i^{\prime}\right.$ and $\left.r^{\prime}\right)$ are the initial (new) set of occupied and virtual orbitals, respectively, and $C_{i r}$ the coefficient of a singly excited determinant $a_{r}^{+} a_{i} \Phi_{0}$. The new set of $i^{\prime}$ and 
$r^{\prime}$ orbitals is then orthogonalized and the method is iterated until convergence is achieved as in the proposed procedure.

\section{Optimization of the localized orbitals: Technical aspects}

The technique that we use to impose the block diagonal shape of the density matrix at each iteration consists of mixing the orbitals belonging to both classes I and V using the exact passage matrix $U_{D}$ that fully diagonalizes the density matrix, while preventing as much as possible the inside block (intraclass) rotations of these orbitals.

The diagonal density matrix $R_{D}$ is obtained by the unitary passage matrix $U_{D}$ according to the equation

$$
R_{D}=U_{D}^{+} R U_{D} .
$$

The matrix $R_{D}$ gives completely delocalized natural orbitals. However, the inside (intrablock) rotations may be approximately calculated by projecting the matrix $U_{D}$ onto the three blocks corresponding to the I and $\mathrm{V}$ subspaces of the density matrix. Since the so-obtained vectors are no more orthogonal inside each block, an $S^{-1 / 2}$ orthogonalization is performed, providing a block diagonal unitary passage matrix $U_{P}$. The application of the matrix $U_{P}$ to the diagonal density matrix leads to a mixing of the vectors inside each subspace that restores the local nature of the initial orbitals. The block diagonal density matrix $R_{D P}$ obeys the equation

$$
R_{D P}=U_{P} R_{D} U_{P}^{+} .
$$

Notice that if $R$ is already block diagonal then $R_{D P}=R$. The $R_{D P}$ matrix might be directly obtained by the unitary passage matrix $W=U_{D} U_{P}^{+}$, so that

$$
R_{D P}=U_{P} R_{D} U_{P}^{+}=U_{P}\left(U_{D}^{+} R U_{D}\right) U_{P}^{+}=W^{+} R W .
$$

This procedure provides a set of orbitals on which the references $\Phi_{I}$ as well as the singly excited determinants $a_{r}^{+} a_{i} \Phi_{I}$ are expanded again and the method is iterated until the convergence is achieved, i.e., the density matrix is stationary and diagonal from one iteration to the other.

\section{Multireference local orbitals}

The iterations proceed through the calculation of the density matrix from a CAS plus singles and the calculation of quasinatural orbitals, as sometimes done in post-CASSCF calculations. ${ }^{28}$

As $\mathrm{SCF}(\mathrm{HF})$ localized orbitals seem to be a rational choice for single reference descriptions, CASSCF localized orbitals would be desirable for multireference ones. In the CIS space used above, the single reference determinant $\Phi_{0}$ is replaced by the CAS or, if we want to use a selected approach, by a subset of the CAS. There are now three classes of orbitals, namely the inactive, active, and virtual (I, A, V).

The guess orbitals are obtained in the same way as in the single reference case. Concerning the active orbitals, they can be atom centered (they allow a VB picture of the CAS function), or bond centered (both bonding and antibonding orbitals must be taken as active).

In the optimization process, there are now three classes and, therefore, during the partial diagonalization of the density matrix, the rotations also will be forbidden among active orbitals. The optimization process is, however, not strictly equivalent. One must notice that, while starting from the single reference SCF determinant $\Phi_{0}$, the CIS does not change the energy at convergence; this is no more true when one starts from a CAS. Even if CASSCF orbitals are used, the weight of some singly excited configurations is not zero, and therefore the diagonalization of the CAS plus singles (CAS-CIS) gives an energy slightly lower than the diagonalization of the CAS (CAS-CI). Only if one performs a contracted CI (taking care of all possible linear dependencies), where

$$
\Psi_{\text {CASCIS-contracted }}=\Psi_{\mathrm{CAS}}+\sum_{i, r} C_{i r} a_{r}^{+} a_{i} \Psi_{\mathrm{CAS}},
$$

and imposes the generalized Brillouin theorem, ${ }^{29,30}$ the two energies would be equal. In that case, the energy would be equal to a standard CASSCF result, and the three spaces corresponding to the I, A, and V classes would be equivalent to those obtained by a standard CASSCF. The inactive (respectively, active, virtual) local orbitals could be obtained by rotations of the CASSCF orbitals of the same class.

The uncontracted alternative used in this work consists of working in the space defined by the references and all the nonidentical singly excited determinants. Let us call $S$ $=\left\{\Phi_{I}\right\}$ the set of the references of the CAS. The optimization of the localized orbitals may therefore be achieved within the space $S^{\prime}$ containing $S$ and all the singly nonredundant excited determinants $a_{r}^{+} a_{i} \Phi_{I}$

$$
S^{\prime}=\left\{\Phi_{I}\right\} \cup\left\{a_{r}^{+} a_{i} \Phi_{i}\right\}
$$

Although the procedure will lead to an energy higher than the CASSCF one, working with such a wave function has two advantages over the previous proposition: (i) the corresponding space is orthogonal; (ii) the wave function incorporates dynamical polarization effects, i.e., instantaneous response of the inactive electrons to the fluctuating field created by the active electrons, while the CASSCF function only takes into account the mean field of the active electrons. One must notice, however, that the contracted and uncontracted schemes give very similar orbitals.

The presented method may easily be generalized to a selected multireference space included in the CAS, the space $S$ containing now the set of the selected references, leading to an approximate MCSCF description. Passing from a CAS to an MR space will be especially beneficial when subsequently calculating the dynamical correlation energy, through an $\mathrm{MR}+\mathrm{SD}$ calculation for instance, the list of singly and doubly excited determinants being significantly reduced.

Notice that the generalization of the previous procedure to state average calculations is straightforward when considering a mean density matrix ${ }^{31}$ (averaged for several states $1,2, \ldots, N)$ of the form

$$
R^{1, \ldots, N}=\sum_{K=1, N} \alpha_{K} R^{K},
$$


where $\alpha_{K}$ is a weight that is adjusted according to the physical problem and $R^{K}$ the density matrix calculated for the state $K$.

\section{APPLICATIONS}

The applications presented in this section will illustrate both the computational benefit of using localized orbitals, through possible reduction of the number of references, and the control of the location of the active orbitals when bond breaking is considered.

\section{A. Study of the ground state and the two first singlet $d \rightarrow \pi^{*}$ excited states of the bichromophoric trans- bicyclo[3.3.0] octane-3,7-dione (BOD) molecule}

The BOD molecule is a bichromophoric molecule constituted of two ketone chromophores connected by a saturated bridge. ${ }^{32}$ In such systems, intramolecular electronic energy transfer occurs between the two chromophoric groups. The excitation energy is transferred from the excited donor chromophore to the ground-state acceptor. This process is involved in photosynthesis, polymer photophysics, and photochemical synthesis and is thus of fundamental importance. The qualitative picture of the intramolecular energy transfer that is commonly used is a two-state model generating a double-well potential energy surface. In this compound, the energy transfer process is known to be nonadiabatic, since the energy difference between the two adiabatic excited states $S_{1}$ and $S_{2}$ involved in the process is very small, resulting from a weak interchromophoric electronic coupling. The determination of this coupling requires an accurate calculation of the energy difference between the two singlet states, therefore, the so-called difference-dedicated configuration interaction DDCI $^{33}$ (CAS-DDCI and MR-DDCI) method has been used. $\mathrm{CAS}+\mathrm{SD}$ and $\mathrm{MR}+\mathrm{SD}$ calculations have also been performed to calculate the energy of the ground state $S_{0}$.

Because of the size of the molecule, and since we were essentially interested in checking the performances of our method at the CAS + SD level, STO-3G basis sets $^{34}$ have been used. The geometries used in our calculations have been fully optimized ${ }^{35}$ for the ground state and for the second excited states (that corresponds to the $S_{1} / S_{2}$ conical intersection) at the $\operatorname{CASSCF}(8,6)$ level in a delocalized description. The canonical active orbitals have the same physical content as the chosen localized active orbitals described in the next paragraph.

In order to choose the correct minimal active space, let us consider the ketone group. The study of the $n \rightarrow \pi^{*}$ excitation requires to take as active the $\pi$ and $\pi^{*}$ orbitals of the $\mathrm{CO}$ bond and the nonbonding $n$ orbital of the oxygen lone pair. Notice that for a better analysis of the physics we have chosen to take two active LMOs $\left(\pi\right.$ and $\left.\pi^{*}\right)$ instead of the two $p$ atomic orbitals. Since the molecule is bichromophoric, one has 8 electrons in 6 orbitals, leading to an active space $[\mathrm{CAS}(8,6)]$ containing 225 references, if no symmetry is imposed.

The optimization of the orbitals has been performed in a different manner for the ground state and for the excited states. The orbitals of the ground state have been optimized
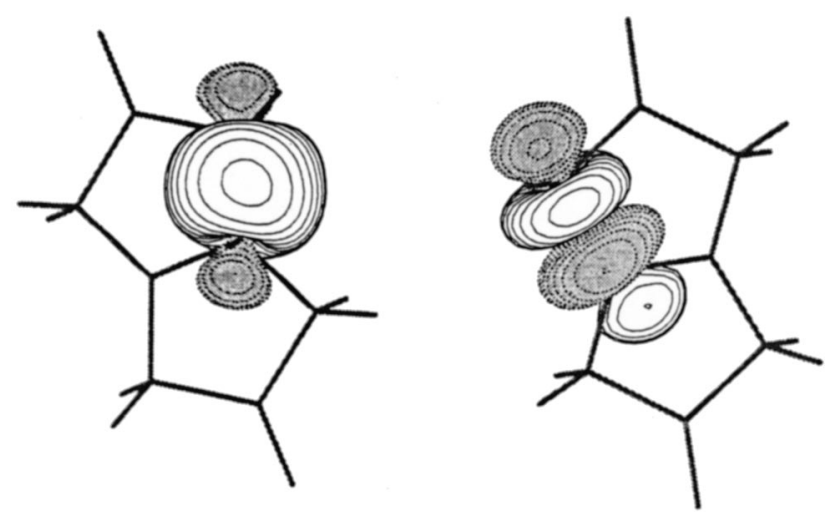

FIG. 1. Example of a bonding (on the left) and an antibonding (on the right) LMO optimized for the ground state of the BOD molecule.

using the density matrix of the ground-state function $\Psi_{\text {CAS }+S}$. Concerning the calculation of the $S_{1}-S_{2}$ energy difference, an orbital optimization using an average density matrix, the mean between the two density matrices of the singlet excited states $\Psi_{\mathrm{CAS}+S}^{S_{1}}$ and $\Psi_{\mathrm{CAS}+S}^{S_{2}}$, was preferable. The so-obtained orbitals are therefore adapted to both excited states, as would be the case for orbitals resulting from an average CASSCF procedure. To exhibit the strongly localized character of the optimized orbitals, we have represented in Fig. 1 a bonding LMO and an antibonding one, optimized for the ground state. Concerning the ground state, it has been possible to restrict the CAS to an MR space containing only the 18 determinants of major weight in the CASCI wave function without affecting the accuracy of the results at the MRCI (diagonalization of the references space) level as well as at the MR+SD level. Comparisons of the obtained results (for truncated and nontruncated spaces) with the delocalized CASSCF and CAS + SD procedures are presented in Table II. The CASCI energies obtained from our procedure are very close to the traditional CASSCF ones, the error being $0.038 \mathrm{eV}$. Reducing the space to $10 \%$ of its full size only affects the result by $0.014 \mathrm{eV}$.

The full CAS + SD space (if one does not use symmetries) contains around $1.4 \times 10^{8}$ determinants and is therefore not tractable. Our calculation at the MR + SD level involves only $13 \times 10^{6}$ determinants and therefore also corresponds to a reduction of the space to $10 \%$ of its full size. In order to check our method, we have compared the obtained groundstate energy with the one resulting from a CAS $+\mathrm{SD}$ calcu-

TABLE II. Ground-state energy of the BOD molecule calculated using the optimized localized orbitals at several level of correlation. The results obtained from delocalized CASSCF and CASSD methods are reported for comparison.

\begin{tabular}{lccc}
\hline \hline & $\begin{array}{c}\text { Dim. of the space } \\
\left(\mathrm{C}_{2 h} \text { symmetry }\right)\end{array}$ & $\begin{array}{c}\text { Dim. without } \\
\text { symmetry }\end{array}$ & Energy (a.u.) \\
\hline CASSCF(deloc.) & & 225 & -452.884129 \\
CAS(CI)(loc.) & & 225 & -452.882729 \\
MR(CI)(loc.) & & 18 & -452.882229 \\
CASSD(deloc.) & $36 \times 10^{6}$ & $1.4 \times 10^{7}$ & -453.342491 \\
MRSD(loc.) & & $13 \times 10^{6}$ & -453.342071 \\
\hline \hline
\end{tabular}


TABLE III. Energy difference between the $S_{1}$ and $S_{2}$ excited states of the BOD molecule calculated using the optimized localized orbitals with the DDCI algorithm. The CASDDCI result obtained using canonical orbitals is reported for comparison.

\begin{tabular}{lcc}
\hline \hline & Dim. of the space & Energy difference (a.u.) \\
\hline CAS(DDCI)(deloc.) & $13.7 \times 10^{6}$ and 225 ref. & $2.33 \times 10^{-4}$ \\
CAS(DDCI)(loc.) & $13.7 \times 10^{6}$ and 225 ref. & $2.4 \times 10^{-4}$ \\
MR(DDCI)(loc.) & $9.6 \times 10^{6}$ and 58 ref. & $2.34 \times 10^{-4}$ \\
\hline
\end{tabular}

lation in canonical orbitals. This last calculation has been performed using a $C_{2 h}$ symmetry. The localized MR+SD results is in excellent agreement with the delocalized CAS $+\mathrm{SD}$ one, the error being only $0.011 \mathrm{eV}$.

The minimal number of references necessary to describe correctly both excited states is 58. Results of the MRDDCI calculations are reported in Table III. This result compares very well with the CASDDCI one showing that such small energy differences can be calculated with an excellent accuracy using this method. One should notice here that the DDCI procedure eliminates all the double excitations which do not touch the active orbitals (keeping only the determinants that play a differential role on the energy difference); this explains why the reduction of the reference space is not linearly correlated with the reduction of the MRDDCI one. Of course, the gain would be more dramatic if traditional $\mathrm{MR}+\mathrm{SD}$ schemes were used.

\section{B. Bond breaking}

In order to show the problems associated with a delocalized CASSCF description, we consider the case of the dissociation of a $\mathrm{C}-\mathrm{H}$ bond in ethylene, which has been the subject of several methodological works. ${ }^{28,33,36}$ We study a "radial" dissociation, in the sense that all the angles of the molecules were kept unchanged during the dissociation pro- cess. We used the MOLCAS package $\mathrm{e}^{37-39}$ and the atomic natural orbitals (ANO) $3 s 2 p 1 d$ basis set of Widmark et al. ${ }^{24}$ The experimental geometry is used for the rest of the molecule $\left(d_{\mathrm{C}-\mathrm{H}}=1.085 \AA, d_{\mathrm{C}-\mathrm{C}}=1.339 \AA, \mathrm{HCH}=124.8\right)$, with ANO basis sets of type $(3 s 2 p 1 d)$ for the $\mathrm{C}$ and $(2 s 1 p)$ for the $\mathrm{H}$ atoms. ${ }^{24}$ For distances larger than $2.085 \AA$ the active orbitals are correctly located on the breaking bond, and a canonical CAS (2/2) calculation gives a curve that is parallel to the valence CAS(12/12) result. At shorter distances, the two active orbitals become $\pi$ and $\sigma^{*}$ in nature, and the energy is consequently too high. Lower solutions exist that can be reached by changing the guess orbitals. In particular, there exists a space $\left(\pi, \pi^{*}\right)$ which gives the lowest energy, but which is not related to the process under consideration. In the present work, for the sake of simplicity, we consider only those solutions that can be obtained from the HOMO/LUMO guess. The usual solution to overcome discontinuities in a CASSCF calculation is to increase the active space size, so we tested a CAS(4/4) space. This improves the situation, but the wave function discontinuity is still present. Moreover, a larger active space means more determinants, and hence a higher computational cost. On the contrary, the use of localized orbitals permits a correct correlation of precisely those orbitals involved in the breaking process. As shown in Fig. 2, the localized CAS(2/2) energy curve is continuous and parallel to the valence CAS(12/12) result.

The discontinuity in the CASSCF results does not disappear at CI level. The CAS+ SD calculations show that it is still present at truncated CI level if delocalized CASSCF orbitals are used (see Fig. 3). It is smaller for the $\operatorname{CAS}(4 / 4)+\mathrm{SD}$ than for the $\operatorname{CAS}(2 / 2)+\mathrm{SD}$, but it is not eliminated. The sizes of the $\operatorname{CAS}(2 / 2)+\mathrm{SD}$ and $\operatorname{CAS}(4 / 4)$ + SD are 637848 and 4962550 determinants, respectively. One may notice in this particular case that, due to the lack of control over the nature of the active orbitals, the delocalized $\mathrm{CAS}(2 / 2)+\mathrm{SD}$ converges, at short distances, on a higher



FIG. 2. C-H bond breaking in ethylene in delocalized and localized description (see the text). 


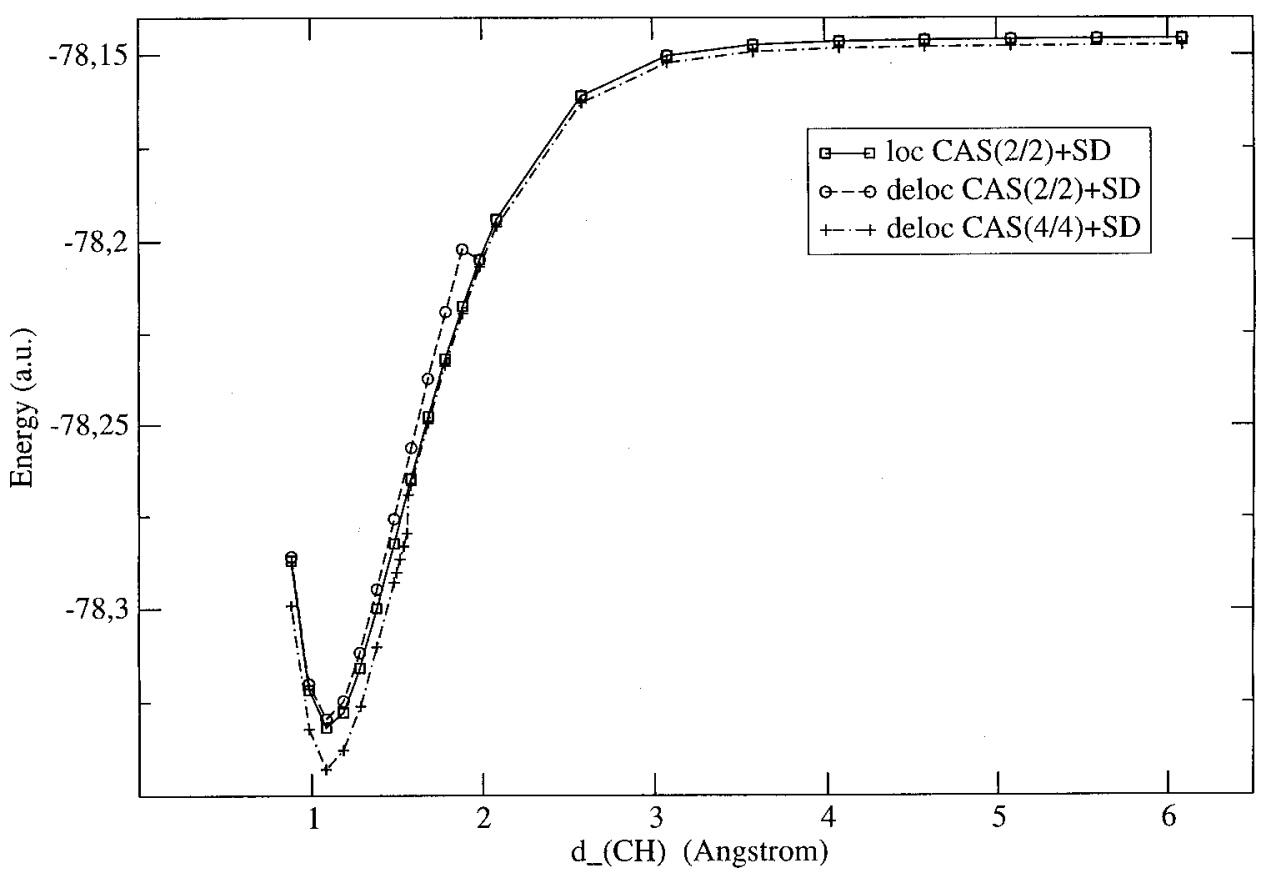

FIG. 3. C-H bond breaking in ethylene in delocalized and localized description for CAS + SD calculations.

energy solution, while the $\mathrm{CAS}(4 / 4)+\mathrm{SD}$ converges to a low energy solution (with the $\pi / \pi^{*}$ orbitals in the active space). The CAS(12/12) $+\mathrm{SD}$ is impossible to perform, since its size is more than $10^{10}$. On the other hand, the calculation with local orbitals does not show any discontinuity even at the $\operatorname{CAS}(2 / 2)+$ s.d. level.

\section{CONCLUSION}

The present work proposes a method to obtain a set of orthogonal localized orbitals within a MRCI calculation. The orbitals can be, on request, atom-, bond- or fragmentcentered, and this gives a great flexibility to the procedure. The algorithm does not proceed through an energy minimization, as CASSCF does. Instead, the orbitals are obtained through the iterative (partial) diagonalization of the oneparticle density matrix obtained from the CI of the single excitations acting on the references. In this way, by starting from a guess of local orbitals, locality is maintained by annihilating, at each iteration, the density matrix off-diagonal blocks only.

The method provides energies and wave functions that are extremely close to those obtained from the corresponding CASSCF calculation having the same active space. Moreover, the exact CASSCF solution is recovered if an internally contracted CI or perturbative scheme is used. The implementation of a contracted perturbative algorithm that produces the exact CASSCF wave function will be presented in a forthcoming paper.

The present procedure is particularly suitable for the treatment of quasidegenerate systems. In particular, it works perfectly well even in those cases where an SCF solution cannot be found, and where SCF-based localization methods (e.g., Boys) are therefore of a difficult application. We notice that the use of localized orbitals in a CASSCF or postCASSCF context is important for several reasons. (i) It gives a fine control on the nature of the active orbitals. In this way, it is possible to include in the active space just those orbitals that are strictly required by the nature of the studied phenomenon. As a consequence, as we have shown in the case of the $\mathrm{C}-\mathrm{H}$ bond breaking in ethylene, a dramatic reduction of the computational effort can be obtained. One should notice that the wave function obtained in such a way for a given active space is independent (at convergence) of orbital localization. This means that if a rotation is performed (within each orbital class) on the localized orbitals, a set of equivalent orbitals is obtained. These equivalent orbitals can be delocalized, but they produce a wave function that is identical to the localized one (the two sets of orbitals give the same energy).

(ii) It permits a rational reduction of the reference number with respect to a CAS expansion even in strongly correlated systems, such as multiple-bond breaking or magnetic systems. The benefit of this reduction is especially important when one treats the dynamical correlation effects through $\mathrm{MR}+\mathrm{SD}$ calculations, the size of which is proportional to the number of references.

(iii) It provides orthogonal localized virtual orbitals which are difficult to obtain in many a posteriori localization procedures.

At the moment, our approach is relatively expensive, since it implies the transformation of the bielectronic integrals at each iteration. However, we notice that only a subset of the two-electron integrals needs to be transformed in order to perform the required single-CI calculation. One could further reduce the number of integrals needed by using a second-order perturbative scheme, involving integrals having at most one nonactive orbital index. A further efficiency aspect is related to the symmetry of the system. The present 
code does not take advantage of the equivalence between the local MOs in symmetric systems. One could use the symmetry properties in the calculation of the integrals as well as in the CI expansion (by considering only the representative determinant of each class of symmetry). Work is in progress in both directions, in order to reduce the cost of the integraltransformation step, and to take advantage of the symmetry of the system.

Finally, this work participates in the general renewal of interest for the localized descriptions, widely motivated by the production of linear-scaling algorithms. In none of the presented applications did we use the local character of the orbitals to neglect small enough integrals. The multireference approach (with respect to single reference) is more complex, and it is probably impossible to reach the impressive systems (thousands of orbitals) the linear scaling calculations deal with. However, we believe that the production of local orbitals in an MR context can represent an important preliminary step toward the use of linear scaling techniques in MR algorithms, allowing us to study much larger systems.

\section{ACKNOWLEDGMENTS}

It is a pleasure to thank Doctor Franck Jolibois for helpful discussions. The Laboratoire de Physique Quantique is UMR of the CNRS (UMR 5626). One of us (C.J.C.) acknowledges the financial support through the TMR activity "Marie Curie Research Training Grants" Grant No. HPMFCT-1999-00285 established by the European Commission.

${ }^{1}$ T. A. Koopmans, Physica (Utrecht) 1, 104 (1933).

${ }^{2}$ J. M. Van Vleck, J. Chem. Phys. 1, 177 (1933); 1, 219 (1933)

${ }^{3}$ Localization and Delocalization in Quantum Chemistry, edited by O. Chalvet, R. Daudel, S. Diner, and J. P. Malrieu (Reidel, Dordrecht, 1975).

${ }^{4}$ L. Brillouin, Act. Sci. Ind., No. 159 (Herrmann, Paris, 1934).

${ }^{5}$ P. Y. Ayala and G. E. Scuseria, J. Chem. Phys. 110, 3660 (1999).

${ }^{6}$ P. Pulay and S. Saebo, in Geometrical Derivatives of Energy Surfaces and Molecular Properties, edited by P. Jorgensen and J. Simons (Reidel, Dordrecht, 1986), p. 95.

${ }^{7}$ M. Schutz, G. Rauhut, and H. J. Werner, J. Comput. Chem. 102, 5997 (1998).

${ }^{8}$ G. Rauhut, P. Pulay, and H. J. Werner, J. Comput. Chem. 19, 1241 (1998).
${ }^{9}$ G. Hetzer, P. Pulay, and H. J. Werner, Chem. Phys. Lett. 290, 143 (1998).

${ }^{10}$ P. Pulay and S. Saebo, Theor. Chim. Acta 69, 357 (1986).

${ }^{11}$ S. Saebo and P. Pulay, J. Chem. Phys. 86, 914 (1987).

${ }^{12}$ C. Hampel and H. J. Werner, J. Chem. Phys. 104, 6286 (1996).

${ }^{13}$ G. E. Scuseria and P. Y. Ayala, J. Chem. Phys. 111, 8330 (1999).

${ }^{14}$ M. Schütz, J. Chem. Phys. 113, 9986 (2002); M. Schütz and H. J. Werner, ibid. 114, 661 (2001).

${ }^{15}$ N. Guihéry, J. P. Malrieu, S. Evangelisti, and D. Maynau, Chem. Phys. Lett. (to be published).

${ }^{16}$ W. J. Hunt, P. J. Hay, and W. A. Goddard, III, J. Chem. Phys. 57, 738 (1972).

${ }^{17}$ S. F. Boys, Rev. Mod. Phys. 32, 2 (1960).

${ }^{18}$ C. Edminston and K. Ruedenberg, Rev. Mod. Phys. 34, 457 (1963).

${ }^{19}$ J. Pipek and P. G. Mezey, J. Chem. Phys. 90, 4916 (1989).

${ }^{20}$ C. Angeli, G. Del Re, and M. Persico, Chem. Phys. Lett. 233, 102 (1995).

${ }^{21}$ W. A. Adams, J. Chem. Phys. 34, 89 (1961).

${ }^{22}$ P. W. Anderson, Phys. Rev. Lett. 4, 17 (1969).

${ }^{23}$ T. L. Gilbert, in Molecular Orbitals in Chemistry, Physics and Biology, edited by P. O. Löwdin and B. Pullman (Academic, New York, 1964), p. 405.

${ }^{24}$ P. O. Widmark, P. A. Malmqvist, and B. Roos, Theor. Chim. Acta 77, 291 (1990).

${ }^{25}$ G. Chambaud, M. Gerard-Ain, E. Kassab, B. Lévy, and P. Pernot, Chem. Phys. 90, 271 (1984).

${ }^{26}$ F. Illas, M. Merchan, M. Pélissier, and J. P. Malrieu, Chem. Phys. 107, 361 (1986).

${ }^{27}$ J. P. Daudey, Chem. Phys. Lett. 24, 574 (1974); J. Rubio, A. Povill, J. P. Malrieu, and P. Reinhardt, J. Chem. Phys. 107, 10044 (1997).

${ }^{28}$ J. Miralles, R. Caballol, and J. P. Malrieu, Chem. Phys. 153, 25 (1991).

${ }^{29}$ B. Levy and G. Berthier, Int. J. Quantum Chem. 2, 307 (1968).

${ }^{30}$ K. Ruedenberg, L. M. Cheung, and T. Elbert, Int. J. Quantum Chem. 16, 1069 (1979).

${ }^{31}$ V. M. Garcia, O. Castell, R. Caballol, and J. P. Malrieu, Chem. Phys. Lett. 238, 222 (1995).

${ }^{32}$ P. H. Schipper and H. P. J. M. Dekkers, J. Am. Chem. Soc. 105, 145 (1983).

${ }^{33}$ J. Miralles, O. Castell, R. Caballol, and J. P. Malrieu, Chem. Phys. 172, 33 (1993).

${ }^{34}$ W. J. Hehre, R. F. Stewart, and J. A. Pople, J. Chem. Phys. 51, 2657 (1969).

${ }^{35}$ F. Jolibois, M. Bearpark, and M. Robb, J. Phys. Chem. A (submitted).

${ }^{36}$ C. J. Wu and E. A. Carter, J. Am. Chem. Soc. 112, 5893 (1990).

${ }^{37}$ K. Anderson, P. Malmqvist, B. O. Roos, A. J. Sadlej, and K. Volensky, J. Phys. Chem. 94, 5483 (1990); K. Anderson, P. Malmqvist, and B. O. Roos, J. Chem. Phys. 96, 1218 (1992).

${ }^{38}$ C. Angeli, R. Cimiraglia, M. Persico, and A. Toniolo, Theor. Chim. Acta 98, 57 (1997).

${ }^{39}$ D. L. Cooper, J. Geratt, and M. Raimondi, Adv. Chem. Phys. 67, 319 (1987). 